\title{
Comparative toxicological assessment of PAMAM and thiophosphoryl dendrimers using embryonic zebrafish
}

This article was published in the following Dove Press journal:

International Journal of Nanomedicine

17 April 2014

Number of times this article has been viewed

\author{
Joseph B Pryor' \\ Bryan J Harper' \\ Stacey L Harper ${ }^{1,2}$ \\ 'Department of Environmental and \\ Molecular Toxicology, Oregon State \\ University, Corvallis, OR, USA; \\ ${ }^{2}$ School of Chemical, Biological, and \\ Environmental Engineering, Oregon \\ State University, Corvallis, OR, USA
}

Correspondence: Stacey L Harper Oregon State University, 1007

Agriculture and Life Sciences Building,

Corvallis, Oregon 9733I, USA

Tel + I 54 I 737279 |

Fax + I 54| 7370497

Email stacey.harper@oregonstate.edu

\begin{abstract}
Dendrimers are well-defined, polymeric nanomaterials currently being investigated for biomedical applications such as medical imaging, gene therapy, and tissue targeted therapy. Initially, higher generation (size) dendrimers were of interest because of their drug carrying capacity. However, increased generation was associated with increased toxicity. The majority of studies exploring dendrimer toxicity have focused on a small range of materials using cell culture methods, with few studies investigating the toxicity across a wide range of materials in vivo. The objective of the present study was to investigate the role of surface charge and generation in dendrimer toxicity using embryonic zebrafish (Danio rerio) as a model vertebrate. Due to the generational and charge effects observed at the cellular level, higher generation cationic dendrimers were hypothesized to be more toxic than lower generation anionic or neutral dendrimers with the same core composition. Polyamidoamine (PAMAM) dendrimers elicited significant morbidity and mortality as generation was decreased. No significant adverse effects were observed from the suite of thiophosphoryl dendrimers studied. Exposure to $\geq 50 \mathrm{ppm}$ cationic PAMAM dendrimers G3-amine, G4-amine, G5-amine, and G6-amine caused 100\% mortality by 24 hours post-fertilization. Cationic PAMAM G6-amine at $250 \mathrm{ppm}$ was found to be statistically more toxic than both neutral PAMAM G6-amidoethanol and anionic PAMAM G6-succinamic acid at the same concentration. The toxicity observed within the suite of varying dendrimers provides evidence that surface charge may be the best indicator of dendrimer toxicity. Dendrimer class and generation are other potential contributors to the toxicity of dendrimers. Further studies are required to better understand the relative role each plays in driving the toxicity of dendrimers. To the best of our knowledge, this is the first in vivo study to address such a broad range of dendrimers.
\end{abstract}

Keywords: nanomaterials, in vivo, toxicity, generation, surface chemistry, zebrafish

\section{Introduction}

The use of polymeric nanomaterials such as dendrimers in the development and delivery of therapeutic compounds has emerged as a promising field of biomedical engineering. ${ }^{1}$ Dendrimers have a well-defined branching architecture consisting of an initiator core, a radiating interior structural layer composed of repeating generations (G0-G10), and terminal functional groups attached to the outermost generation (Figure 1). ${ }^{2,3}$ Subsequent generations increase in diameter, the number of terminal functional groups, and the number of vacancies available to carry therapeutics. ${ }^{4-7}$ The low polydispersity index and the ability to precisely control their surface chemistry make dendrimers useful for medical imaging, enhancing the solubility and/or bioavailability of drugs, brain-target drug therapy, and specific targeting mechanism for anti-cancer 


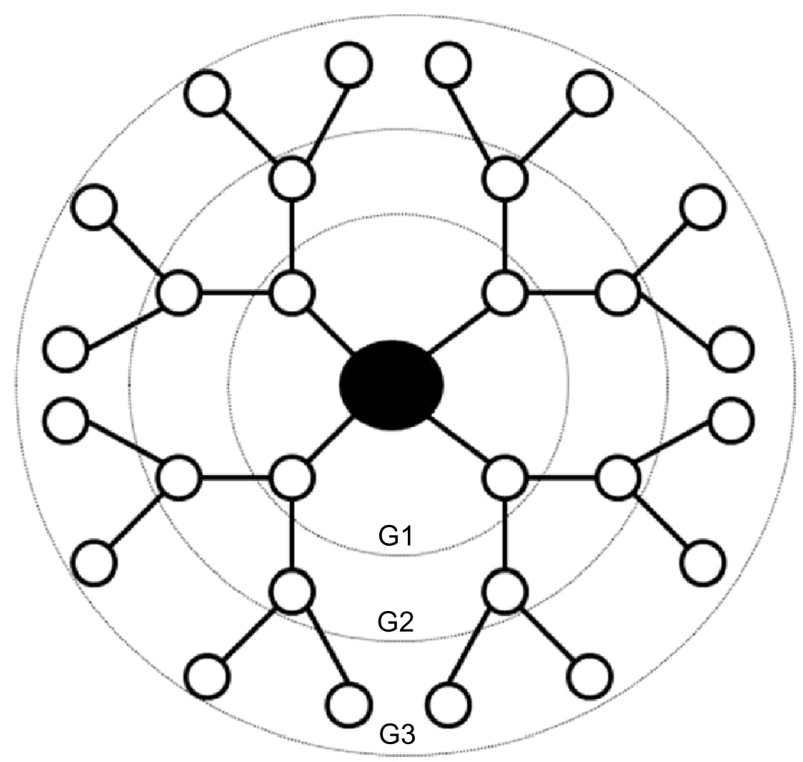

Figure I Generation $(G) 3$ dendrimer architecture.

Note: Dendrimers consist of core, interior branching, and terminal functional groups.

therapeutics and gene therapy. ${ }^{8-10}$ The stability, precise size, and defined chemistry of dendrimers also make them ideal for the development of structure-property relationships.

Despite the myriad of potential biomedical applications of dendrimers, previous research exploring the toxicity and biocompatibility of dendritic therapeutics suggests some dendrimers may display inherent toxicity attributed to a combination of generation and charge. ${ }^{11-14}$ Although the mechanism explaining generational effects is still unclear, higher generation dendrimers have been observed to cause nanoscale holes in the lipid bilayer, resulting in more toxicity while other authors have found that increasing generation attenuates toxicity, ${ }^{13-15}$ especially for very high generation dendrimers, perhaps due to conformational changes. ${ }^{11,16-18}$ Regardless of dendrimer generation, some cationic dendrimers have been shown to interact with negatively charged cell membranes. ${ }^{19}$ An increasing number of studies in diverse cell lines have shown that exposure to cationic dendrimers results in cell membrane permeability, cellular lysis, and cytotoxicity., ${ }^{90-23}$ Recent studies in mice and zebrafish (Danio rerio) have also shown polyamidoamine (PAMAM) dendrimers with amine terminated groups affected blood-clot formation when administered intravenously, while neutral and anionic dendrimers of the same generation demonstrated no observable vascular complications. ${ }^{24,25}$ The authors suggest that these effects are driven by the high affinity amine for platelets or vascular endothelium. ${ }^{24}$ The observed toxicity with dendrimers however, is likely due to a combination of generation and charge, as the net charge is dependent on the generation. ${ }^{11,18}$ In contrast with PAMAM dendrimers, the limited research on thiophosphoryl dendrimers has demonstrated minimal inherent toxicity in vitro. ${ }^{26}$

The majority of studies to date have focused on dendrimer toxicity in cell culture, ${ }^{20}$ with fewer studies investigating the toxicity in vivo. Dendrimer toxicity was explored using several PAMAM (Figure 2) and thiophosphoryl (Figure 3) dendrimers that varied in generation and charge in embryonic zebrafish (D. rerio). Due to both the generational and cationic toxicity observed at the cellular level, higher generation cationic dendrimers were hypothesized to be more reactive and thus more toxic than lower generation anionic or neutral dendrimers with the same core composition. ${ }^{11,16-18}$ Zebrafish were selected as a model organism due to their similarities in anatomy and physiology, cell structure, and signaling process to other vertebrates and their tissue clarity which allows for visual inspection of most systems during development. In addition, the rapid development of zebrafish from a few cells to fully functioning juveniles by 120 hours post-fertilization (hpf) allows for high-throughput experimental designs at relatively low cost. ${ }^{27-29}$ The nature of biomedical applications associated with dendrimers makes understanding how their biological interactions relate to structural features, such as generation and charge, an important step in designing safer and more efficient nanopharmaceuticals.

\section{Materials and methods Dendrimers}

Thiophosphoryl-phenoxymethyl(methylhydrazono) 0.5 Generation $(\mathrm{G})$ and $1.5 \mathrm{G}$ dendrimers were purchased from Fisher Scientific (Fair Lawn, NJ, USA). The 2.5G, 3.5G, and $5 \mathrm{G}$ were purchased from Sigma-Aldrich (St Louis, MO, USA). All PAMAM dendrimers were commercially purchased through Dendritic Nanotechnologies Inc. (Mount Pleasant, MI, USA). Dry dendrimers were stored as shipped at $25^{\circ} \mathrm{C}$ while PAMAM G5-succinamic acid was shipped and stored in solution at $-20^{\circ} \mathrm{C}$; all dendrimers were tested without further modification or purification. Detailed core composition, generation, surface chemistry, and surface charges of the dendrimers used in this study are listed in Table 1.

\section{Dendrimer preparation}

Dendrimer stock solutions were prepared at $1,000 \mathrm{mg} / \mathrm{L}$ in embryo media on the day of the exposure and vortexed for 1-2 minutes to ensure uniform dispersion. An hour prior to exposures, 5-fold serial dilutions were conducted with embryo media to make exposure concentrations from 0.016 to 


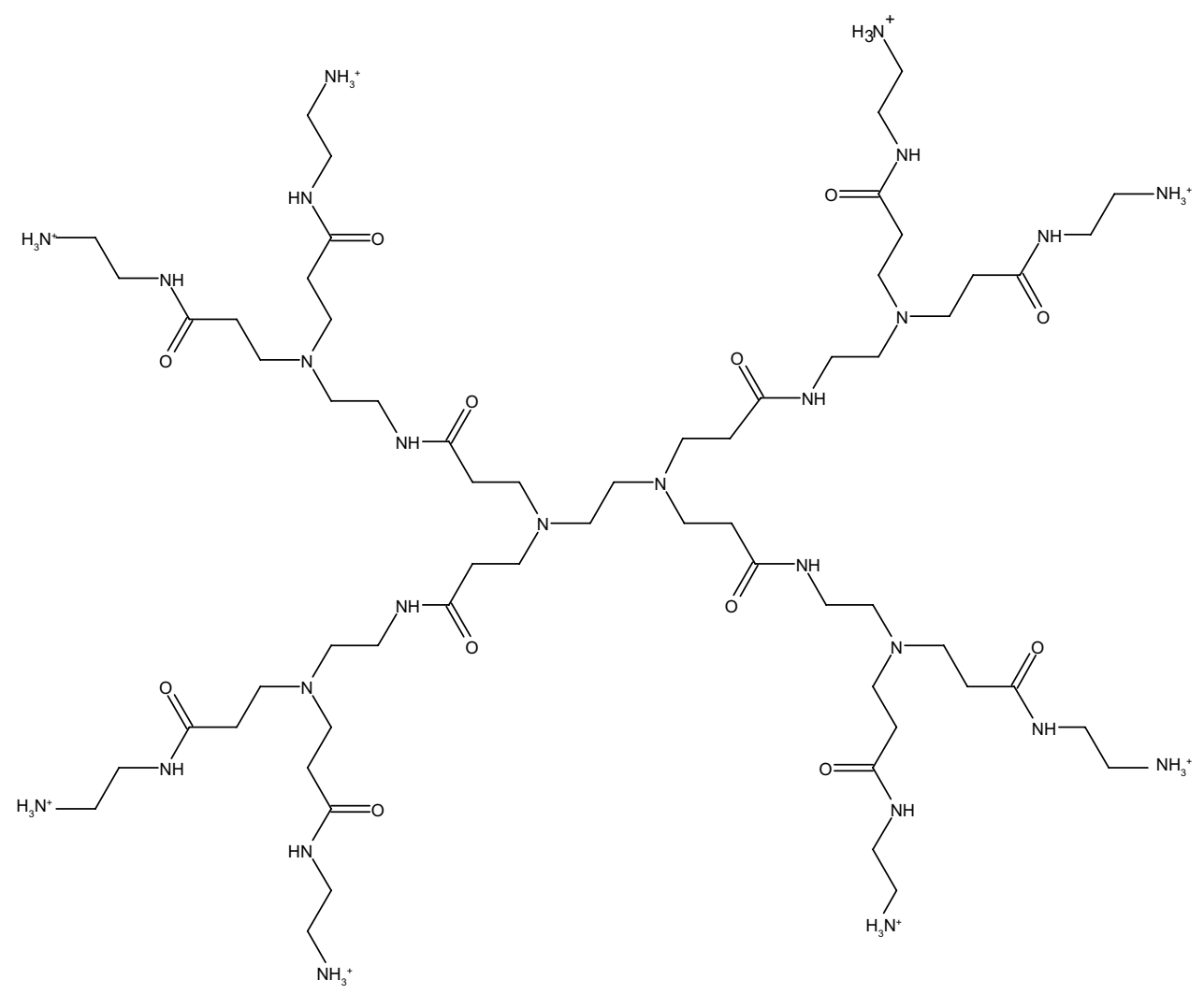

Figure 2 Chemical structure of generation I polyamidoamine (PAMAM) dendrimer.

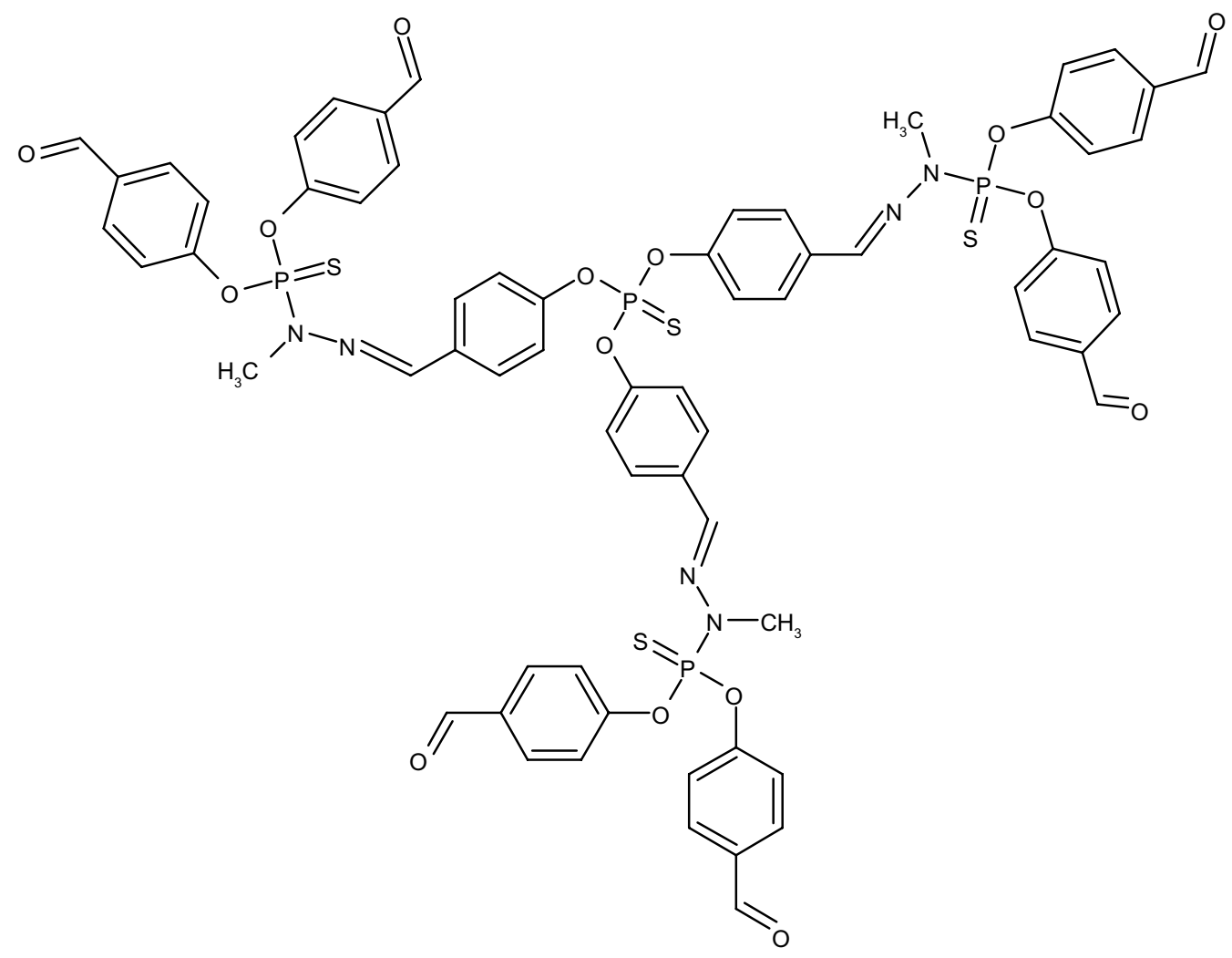

Figure 3 Chemical structure of generation 1.5 thiophosphoryl dendrimer. 
Table I Physical characteristics of polyamidoamine (PAMAM) and thiophosphoryl dendrimers

\begin{tabular}{|c|c|c|c|c|c|}
\hline Name & Core composition & Generation & Surface group & Charge & $\begin{array}{l}\text { Number of } \\
\text { surface groups }\end{array}$ \\
\hline PAMAM G3-amine & I,4-diaminobutane & 3 & Amine & + & 32 \\
\hline PAMAM G4-amine & I,4-diaminobutane & 4 & Amine & + & 64 \\
\hline PAMAM G5-amine & I,4-diaminobutane & 5 & Amine & + & 128 \\
\hline PAMAM G6-amine & I,4-diaminobutane & 6 & Amine & + & 256 \\
\hline PAMAM G6-succinamic acid & I,4-diaminobutane & 6 & Succinamic acid & - & 256 \\
\hline PAMAM G5-succinamic acid & I,4-diaminobutane & 5 & Succinamic acid & - & 128 \\
\hline PAMAM G6-amidoethanol & I,4-diaminobutane & 6 & Amidoethanol & 0 & 256 \\
\hline Thio G0.5-aldehyde & Thiophosphoryl & 0.5 & Aldehyde & 0 & 3 \\
\hline Thio GI.5-aldehyde & Thiophosphoryl & 1.5 & Aldehyde & 0 & 6 \\
\hline Thio G2.5-aldehyde & Thiophosphoryl & 2.5 & Aldehyde & 0 & 12 \\
\hline Thio G3.5-aldehyde & Thiophosphoryl & 3.5 & Aldehyde & 0 & 24 \\
\hline Thio G5-DPT & Thiophosphoryl & 5 & Dichlorophosphinothioyl & 0 & 48 \\
\hline
\end{tabular}

$250 \mathrm{mg} / \mathrm{L}$. Concentrations above $250 \mathrm{mg} / \mathrm{L}$ were considered outside the realistic exposure dose. Dilutions were stored in an incubator at $26.9^{\circ} \mathrm{C}$ until the exposure.

\section{Zebrafish exposure}

Zebrafish (D. rerio) embryos were collected from group spawns of wild-type D5 fish housed at the Sinnhuber Aquatic Research Laboratory (Oregon State University, Corvallis, OR, USA) and staged such that the chorion surrounding the embryo could be removed enzymatically at $6 \mathrm{hpf.} .^{30}$ Dechorionation removes the barrier provided by the chorion in an effort to increase the bioavailability of the particle interacting with the embryo. A $0.6 \%$ Instant Ocean ${ }^{\circledR}$ salt solution (Aquatic Ecosystems, Apopka, FL, USA) was added to deionized water to make embryo media. Embryo media was prepared and adjusted with sodium bicarbonate until a $\mathrm{pH}$ of 7.0-7.4 and a conductivity of $480-600 \mu \mathrm{s} / \mathrm{cm}$ was obtained. Newly fertilized embryos were collected and staged to ensure the zebrafish were treated at the same developmental stage. ${ }^{31}$ Zebrafish were kept at $26.9^{\circ} \mathrm{C}$ on a $14 \mathrm{hour}(\mathrm{hr}) / 10 \mathrm{hr}$ light/dark cycle. At $8 \mathrm{hpf}$, a total of 24 embryos per treatment were exposed individually in 96 well plates to 5 -fold serial dilutions of dendrimers $(0.016,0.08,0.4,2,10,50,250 \mathrm{ppm})$ or embryo media alone (control). At $24 \mathrm{hpf}$, embryos were assessed for mortality, developmental progression, notochord malformation, and spontaneous movement. At $120 \mathrm{hpf}$, embryos were assessed for mortality, as well as behavioral, and physical malformations such as: axis, brain, circulation, eye, caudal fin, pectoral fin, jaw, otic, pigment, pericardial edema, yolk sac edema, snout, swim bladder, trunk, somite, and a touch response according to previously published methods. ${ }^{32,33}$

Data for individual endpoints analyzed here are freely available on the open-source Nanomaterial-Biological Interactions Knowledgebase (http://www.nbi.oregonstate.edu).

\section{Statistical analysis}

Experimental replicates were analyzed using one way analysis of variance (ANOVA) to ensure replicates were not statistically different from one another. ANOVA was also used to compare mortality between the PAMAM and thiophosphoryl dendrimer groups. The Fisher's exact test was used to determine if individual sublethal responses differed significantly from control. Spearman's rank order was used to correlate percent mortality with dosages. All statistical analysis was performed using Sigmaplot version 12.3 (San Jose, CA, USA) with a significance threshold of $P \leq 0.05$.

\section{Results}

\section{Generation (size) effects}

Identical dendrimers of different generations were compared to explore the role of generation on toxicity. PAMAM and thiophosphoryl dendrimers contained a generational series of four dendrimers each. PAMAM dendrimers elicited significant morbidity and mortality as generation decreased. In order to compare the PAMAM generations, the median lethal concentration $\left(\mathrm{LC}_{50}\right)$ was estimated through linear interpolation (Figure 4). As shown in Table 2, the $\mathrm{LC}_{50}$ of cationic PAMAM dendrimers increased from $2 \mathrm{ppm}$ for $\mathrm{G} 3$ PAMAM-amine to $18 \mathrm{ppm}$ for G6 PAMAM-amine dendrimers. Thiophosphoryl dendrimers induced no significant morbidity or mortality at any concentration tested $\left(\mathrm{LC}_{50}>\right.$ $250 \mathrm{ppm})$.

\section{Surface charge}

The toxicity of PAMAM dendrimers was assessed at 24 and 120 hpf by evaluating concentration effects on embryonic zebrafish mortality, development, and malformations. Neutral thiophosphoryl dendrimers exhibited no observable malformations at any dose throughout the exposure. Exposure 

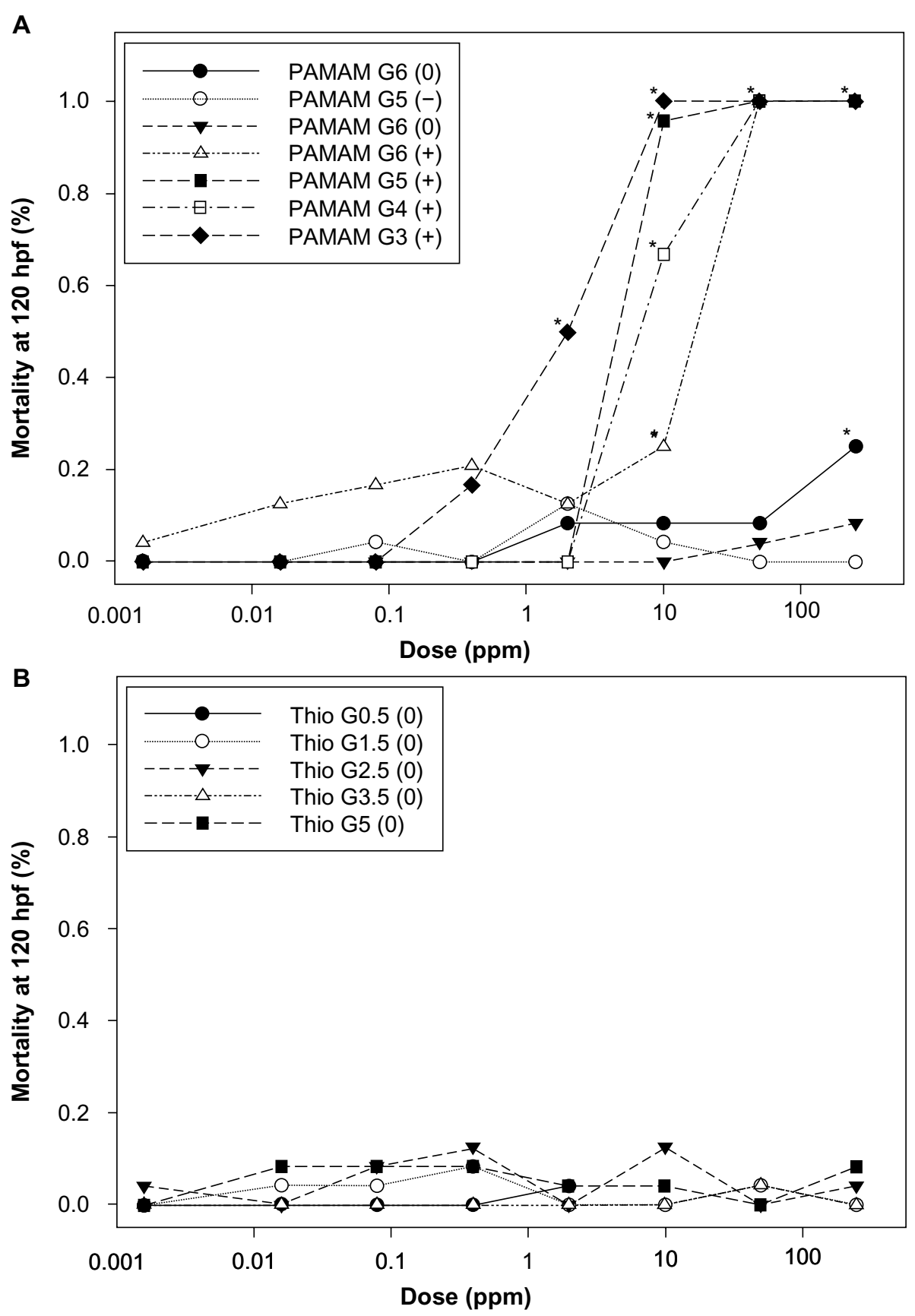

Figure 4 Concentration-response curves for embryonic zebrafish exposed to suites of PAMAM and thiophosphoryl dendrimers.

Note: Concentration-response curves for embryonic zebrafish exposed to suites of PAMAM (A) and thiophosphoryl (B) dendrimers varying in gereation (size), charge and class. Controls contained fishwater only. *denotes significance from control $(P \leq 0.05)$.

Abbreviations: G, generation; Thio, thiophosphoryl; PAMAM, polyamidoamine.

to $\geq 50 \mathrm{ppm}$ cationic PAMAM dendrimers G3-amine, G4-amine, G5-amine, and G6-amine caused 100\% mortality by 24 hpf. Significant cardiac impacts (pericardial edema) were observed at $10 \mathrm{ppm}$ for cationic PAMAM G4-amine and neutral PAMAM G6-amidoethanol $(P<0.05)$. However, negatively charged succinamic acid G5 and G6 dendrimers did not elicit any significant adverse effects even at the highest concentration tested. No other assessed endpoints were significant for either type of dendrimer at any dose or generation.

G6 PAMAM dendrimers that differed in surface chemistry and charge were compared for total mortality at $250 \mathrm{ppm}$. Cationic PAMAM G6-amine at $250 \mathrm{ppm}$ was statistically more toxic than both neutral PAMAM G6-amidoethanol and anionic PAMAM G6-succinamic acid at the same concentration (Figure 5) $(P<0.05)$. At $250 \mathrm{ppm}$, 
Table 2 LC $_{50}$ concentrations calculated through interpolation of mortality across 8 dosages in embryonic zebrafish

\begin{tabular}{ll}
\hline Dendrimer & LC $_{50}(\mathbf{p p m})$ \\
\hline PAMAM G3-amine & 2 \\
PAMAM G4-amine & 6.4 \\
PAMAM G5-amine & 4.5 \\
PAMAM G6-amine & 18 \\
Thio G0.5-aldehyde & $>250$ \\
Thio GI.5-aldehyde & $>250$ \\
Thio G2.5-aldehyde & $>250$ \\
Thio G3.5-aldehyde & $>250$ \\
\hline
\end{tabular}

Note: $L_{50}$ values were only calculated for dendrimers in a generational suite. Abbreviations: G, generation; Thio, thiophosphoryl; PAMAM, polyamidoamine; $\mathrm{LC}_{50}$, median lethal concentration.

$100 \%$ mortality was observed in the PAMAM G6-amine at $250 \mathrm{ppm}$, compared to less than $25 \%$ mortality in the neutral and anionic dendrimers.

\section{PAMAM versus thiophosphoryl}

PAMAM dendrimers as a class were found to be significantly more toxic than thiophosphoryl dendrimers $(P<0.05)$ (Figure 4). In addition, all PAMAM except G5-succinamic acid dendrimer were found to have a statistically significant correlation between the percent mortality and dose (Table S1). PAMAM G6-amidoethanol was found to be statistically more toxic than thiophosphoryl G0.5, G1.5, and G3.5 $(P<0.05)$.

\section{Discussion}

Dendrimers demonstrate generation and charge-dependent toxicity in multiple cell lines, ${ }^{20-22}$ yet little work has been done to investigate effects in vivo. The suite of dendrimers investigated in this study allowed for comparative toxicological analysis between generation, charge, and dendrimer class as possible factors driving toxicity in an in vivo embryo model. Mortality and cardiac impacts (pericardial edema) were sig-

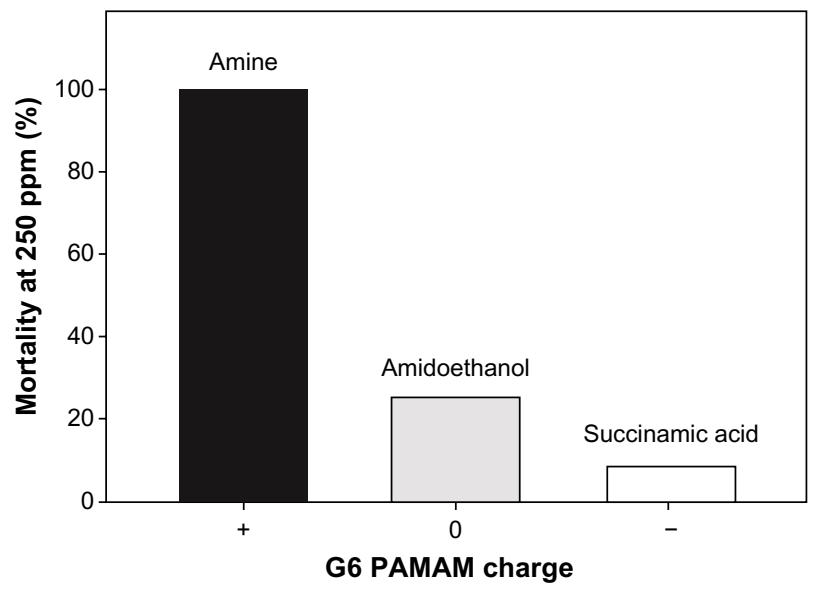

Figure $\mathbf{5}$ Impact of surface chemistry and charge on zebrafish mortality following exposure to 250 ppm G6 PAMAM dendrimer.

Abbreviations: G, generation; PAMAM, polyamidoamine. nificantly increased by higher generation PAMAM dendrimers (Figure 4). Pericardial edema is not necessarily a predicator of any specific pathway being triggered but rather an early stage stressor. ${ }^{34}$ Pericardial edema in zebrafish has been associated with exposure to a wide variety of toxicants including polycyclic aromatic hydrocarbons (PAHs),${ }^{35}$ dioxins, ${ }^{36}$ and gasoline oxygenates. ${ }^{37}$ The PAMAM dendrimers had relatively high toxicity in comparison to the thiophosphoryl dendrimers, indicating higher risk for the combination of surface chemistry and generation studied here. Significant toxicity was only observed with the PAMAM dendrimers, while thiophosphoryl dendrimers induced very little morbidity or mortality at the concentrations tested (0.016-250 ppm).

The results observed for PAMAM and thiophosphoryl dendrimers do not follow the trends previously observed in cell culture studies. ${ }^{16,20-23}$ Although some in vitro studies found an increase in dendrimer generation to be associated with an increase in toxicity, the data presented in this study did not corroborate these findings in an in vivo model at generations tested. A potential explanation is that the largest dendrimers tested in this study were PAMAM G6 and thiophosphoryl G5. In another study, it was found that only PAMAM dendrimers at the G7 level induced 15-40 nm holes in the lipid bilayer. ${ }^{38}$ The proposed mechanism was through the removal and formation of dendrimer filled lipid vesicles. Smaller generation PAMAM dendrimers; however, did not display similar membrane disruptive potential.

Within the suite of five thiophosphoryl dendrimers, increasing generation resulted in no observable increase in toxicity up to the highest dose tested (Table 2). While PAMAM dendrimers demonstrated a generational effect, an increase in generation correlated to a decrease in toxicity (Table 2) similar to previously published studies. ${ }^{15}$ This is perhaps due to a lower bioavailability to the whole organism compared to cells in culture or the stability of higher generation dendrimers in embryo media. The steric hindrance of higher generation dendrimers is thought to make them more likely to change conformation and thus become less stable. ${ }^{39,40}$ In vitro studies ensure the nanoparticle interacts with the cell membrane of a specific cell type. In contrast, the waterborne exposures used in the embryonic zebrafish assay do not indicate a direct dose, rather an exposure concentration, and the impact of surface chemistry and generational size on bioavailability is still unknown.

Cellular uptake of nanoparticles is influenced by their size, ${ }^{41}$ shape, surface charge, and hydrophobicity. ${ }^{42}$ Differential cellular uptake between neutral and anionic surface chemistry 
is unclear. $^{43}$ In similar studies, it was found that anionic dendrimers were more toxic than neutral particles, ${ }^{43}$ but no significant difference between the two were observed here. Despite these contradictions, cationic dendrimers were consistently more toxic than anionic or neutral particles in this study (Figure 5) and other studies..$^{25,43,15}$ The positive charge associated with PAMAM dendrimers allows for interactions with important biomolecules such as DNA, lipid membranes, and mitochondria. Considerable research has demonstrated that cationic nanoparticles induce cellular uptake, ${ }^{42}$ indicating cellular uptake may be the driving force behind the toxicity associated with cationic dendrimers. Manipulation of the terminal amine to another functional group ${ }^{44}$ or methylation of the terminal amine has been shown to decrease toxicity, likely due to a shielding of the cationic charge on the surface. ${ }^{45}$ The toxicity observed among the suite of PAMAM dendrimers supports the hypothesis that surface charge drives the uptake and thus toxicity of dendrimers in vivo. Future studies should focus on quantifying uptake and understanding how changes to the inherent features of dendrimers affects their biodistribution within organisms.

Dendrimer class also plays a role in toxicity with PAMAM and thiophosphoryl dendrimers displaying significant differences in toxicity. Neutral PAMAM G6-amindoethanol exhibited significantly higher toxicity than three of the thiophosphoryl dendrimers, thereby, demonstrating the inherent toxicity of PAMAM dendrimers. PAMAM dendrimers have been shown to cause instability in lipid bilayers, ${ }^{19,38}$ mitochondria, ${ }^{46,47}$ and proteins. ${ }^{48}$ Visual evidence showed that PAMAM dendrimers were transported into cells through endocytosis. ${ }^{49}$ Furthermore, evidence suggests cationic PAMAM dendrimers induce lyosomal instability through alkalinization..$^{50}$ The instability is likely due to a combination of both an increase in $\mathrm{pH}$ as well as a lipid bilayer instability through nanoscale hole formation. ${ }^{19}$ Smaller PAMAM dendrimers (G1-G5) have been shown to prefer water (plasma), whereas higher generation (G6 and G8) dendrimers were demonstrated to partition at the octanol/water interface. ${ }^{51}$ In combination with multiple studies demonstrating the lipid altering capacity of dendrimers, ${ }^{52-54}$ PAMAM dendrimers are anticipated to enter the plasma and result in cardiac malformations. In contrast, the surface groups of thiophosphoryl dendrimers are aldehydes, which have been shown to increase erythrocyte membrane stability and thermal durability, potentially leading to hepatic toxicity. ${ }^{26}$ PAMAM degradation is an area with limited research that could potentially help elucidate one mechanism of toxicity. PAMAM dendrimers contained multiple amide bonds, which are subject to degradation into subunits of ethylenediamine (EDA). If metabolism of PAMAM plays any role in driving the toxicity of this class, ${ }^{55}$ then an increase in generation would result in an EDA increase, suggesting generation is still an important variable to consider in dendrimer toxicity. Future studies should investigate the toxicity of dendrons versus parent dendrimers as well as studying transformations of dendrimer platforms associated with in vivo metabolism and excretion.

The clearly defined structure and amenability of dendrimers makes them ideal for looking at structure-activity relationships. These relationships can then be used in lieu of empirical data for predicting nanomaterial hazards. Principle characteristics that contribute to the inherent toxicity must be revealed in order to minimize the exposure and potential adverse impacts on living organisms. One way to elucidate these characteristics is through rapid, high-throughput assays. Rapid assays such as the one used in this study allow for accurate reliable data that can test a wide range of materials. The rapid assay allowed for a thorough quick assessment of a wide range of dendrimers and to the best of our knowledge, this is the first in vivo study to address such a broad range of dendrimers.

The mechanisms by which dendrimers interact with biological systems are still not fully understood; however, it has been clearly demonstrated that minor alterations in surface chemistry can be used to modify dendrimer toxicity. ${ }^{45}$ Due to the inability to isolate size (generation) from surface charge in this study, generational toxicity is still under investigation. Surface charge was found to be the most effective indicator of relative toxicity in this study; however, core composition also clearly plays a role. Even though it is important to understand how surface chemistry modifications alter dendrimer toxicity, understanding the inherent features of the various dendrimer platforms that drive toxicity provides information on which we can base the safe biological and therapeutic development of future dendrimers. The results presented herein help to elucidate the important role of dendrimer charge and class for understanding and mitigating the toxicity of dendrimers for biomedical applications.

\section{Acknowledgments}

This project was partially funded by National Institutes of Health grants ES017552-01A2, ES016896-01, P30 ES03850. Oregon State University also provided support for JP through the following programs: Undergraduate Research, Innovation Scholarship and Creativity; Honors Experience Scholarship; Honors Promising Finishing Scholarship; and 
Summer Undergraduate Research Experience. The authors would like to thank Dr Josephine Bonventre for manuscript editing.

\section{Disclosure}

The authors report no conflicts of interest in this work.

\section{References}

1. Pignatello R, Musumeci T, Impallomeni G, Carnemolla GM, Puglisi G, Ballistreri A. Poly(3-hydroxybutyrate-co-e-caprolactone) copolymers and poly(3-hydroxybutyrate-co-3-hydroxyvalerate-co-e-caprolactone) terpolymers as novel materials for colloidal drug delivery systems. EuroJ Pharma Sci. 2009;37(3-4):451-462.

2. Ulaszewska MM, Hernando MD, Moreno AU, García AV, Calvo EG, Fernández-Alba AR. Identification and quantification of poly(amidoamine) PAMAM dendrimers of generations 0 to 3 by liquid chromatography/ hybrid quadrupole time-of-flight mass spectrometry in aqueous medium. Rapid Commun Mass Spectrom. 2013;27(7):747-762.

3. Frechet JM. Dendrimers and supramolecular chemistry. Proceedings of the National Academy of Sciences of the United States of America. 2002;99(8):4782-4787.

4. Tomalia DA. Dendrimers as multi-purpose nanodevices for oncology drug delivery and diagnostic imaging. Nanomedicine. 2006;2(4):309.

5. Tomalia DA, Naylor AM, Goddard WA. Starburst dendrimers: molecular-level control of size, shape, surface chemistry, topology, and flexibility from atoms to macroscopic matter. Angew Chem Int Ed Engl. 1990;29(2):138-175.

6. Svenson S, Tomalia DA. Dendrimers in biomedical applications - reflections on the field. Adv Drug Deliv Rev. 2005;57(15):2106-2129.

7. Boas U, Heegaard PMH. Dendrimers in drug research. Chem Soc Rev. 2004;33(1):43-63.

8. Islam MT, Shi X, Balogh L, Baker JR. HPLC separation of different generations of poly(amidoamine) dendrimers modified with various terminal groups. Anal Chemy. 2005;77(7):2063-2070.

9. Nyitrai G, Heja L, Jablonkai I, Pal I, Visy J, Kardos J. Polyamidoamine dendrimer impairs mitochondrial oxidation in brain tissue. J Nanobiotechnology. 2013;11(1):9.

10. Tang MX, Redemann CT, Szoka FC. In vitro gene delivery by degraded polyamidoamine dendrimers. BioconjugChem. 1996;7(6):703-714.

11. Roberts JC, Bhalgat MK, Zera RT. Preliminary biological evaluation of polyamidoamine (PAMAM) StarburstTM dendrimers. $J$ Biomedl Maters Res. 1996;30(1):53-65.

12. Shah N, Steptoe RJ, Parekh HS. Low-generation asymmetric dendrimers exhibit minimal toxicity and effectively complex DNA. J Pept Sci. 2011;17(6):470-478.

13. Naha PC, Davoren M, Casey A, Byrne HJ. An ecotoxicological study of poly(amidoamine) dendrimers-toward quantitative structure activity relationships. Environ Sci Technol. 2009;43(17):6864-6869.

14. Naha PC, Byrne HJ. Generation of intracellular reactive oxygen species and genotoxicity effect to exposure of nanosized polyamidoamine (PAMAM) dendrimers in PLHC-1 cells in vitro. Aquatic Toxicol. 2013;132-133:61-72.

15. Heiden TC, Dengler E, Kao WJ, Heideman W, Peterson RE. Developmental toxicity of low generation PAMAM dendrimers in zebrafish. Toxicol Appl Pharmacol. 2007;225(1):70-79.

16. Tajarobi F, El-Sayed M, Rege BD, Polli JE, Ghandehari H. Transport of poly amidoamine dendrimers across Madin-Darby canine kidney cells. Int J Pharm. 2001;215(1-2):263-267.

17. Naha PC, Davoren M, Lyng FM, Byrne HJ. Reactive oxygen species (ROS) induced cytokine production and cytotoxicity of PAMAM dendrimers in J774A.1 cells. Toxicol Appl Pharmacol. 2010;246(1-2):91-99.

18. Jevprasesphant R, Penny J, Attwood D, McKeown N, D'Emanuele A. Engineering of dendrimer surfaces to enhance transepithelial transport and reduce cytotoxicity. Pharm Res. 2003;20(10):1543-1550.
19. Hong $\mathrm{S}$, Leroueil $\mathrm{P}$, Janus $\mathrm{E}$, et al. Interaction of polycationic polymers with supported lipid bilayers and cells: nanoscale hole formation and enhanced membrane permeability. Bioconjug Chem. 2006;17: $728-734$.

20. Jain K, Kesharwani P, Gupta U, Jain NK. Dendrimer toxicity: Let's meet the challenge. Int J Pharm. 2010;394(1-2):122-142.

21. Sebestik J, Niederhafner P, Jezek J. Peptide and glycopeptide dendrimers and analogous dendrimeric structures and their biomedical applications. Amino Acids. 2011;40(2):301-370.

22. Chen H-T, Neerman MF, Parrish AR, Simanek EE. Cytotoxicity, hemolysis, and acute in vivo toxicity of dendrimers based on melamine, candidate vehicles for drug delivery. J Am Chem Soc. 2004;126(32): 10044-10048.

23. Kolhatkar RB, Kitchens KM, Swaan PW, Ghandehari H. Surface acetylation of polyamidoamine (PAMAM) dendrimers decreases cytotoxicity while maintaining membrane permeability. Bioconjug Chem. 2007;18(6):2054-2060.

24. Jones CF, Campbell RA, Brooks AE, et al. Cationic PAMAM dendrimers aggressively initiate blood clot formation. ACS Nano. 2012;6(11):9900-9910.

25. Dobrovolskaia MA, Patri AK, Simak J, et al. Nanoparticle size and surface charge determine effects of PAMAM dendrimers on human platelets in vitro. Mol Pharm. 2011;9(3):382-393.

26. Domański DM, Bryszewska M, Salamończyk G. Preliminary evaluation of the behavior of fifth-generation thiophosphate dendrimer in biological systems. Biomacromolecules. 2004;5(5):2007-2012.

27. Hill AJ, Teraoka H, Heideman W, Peterson RE. Zebrafish as a model vertebrate for investigating chemical toxicity. Toxicol Sci. 2005;86(1): 6-19.

28. Zon LI, Peterson RT. In vivo drug discovery in the zebrafish. Nat Rev Drug Discov. 2005;4(1):35-44.

29. Truong L, Reif DM, St Mary L, Geier MC, Truong HD, Tanguay RL. Multidimensional in vivo hazard assessment using zebrafish. Toxicol Sci. 2014;137(1):212-233.

30. Usenko CY, Harper SL, Tanguay RL. In vivo evaluation of carbon fullerene toxicity using embryonic zebrafish. Carbon NY. 2007;45(9): 1891-1898.

31. Kimmel CB, Ballard WW, Kimmel SR, Ullmann B, Schilling TF. Stages of embryonic development of the zebrafish. Developmental Dynamics. 1995;203(3):253-310.

32. Harper SL, Carriere JL, Miller JM, Hutchison JE, Maddux BLS, Tanguay RL. Systematic evaluation of nanomaterial toxicity: utility of standardized materials and rapid assays. ACS Nano. 2011;5(6): $4688-4697$.

33. Truong L, Harper S, Tanguay R. Evaluation of embryotoxicity using the zebrafish model. In: Gautier J-C, editor. Drug Safety Evaluation. Vol 691: Humana Press; New York, NY. 2011:271-279.

34. Chen J. Impaired cardiovascular function caused by different stressors elicits a common pathological and transcriptional response in zebrafish embryos. Zebrafish. 2013;10(3):389-400.

35. Incardona JP, Collier TK, Scholz NL. Defects in cardiac function precede morphological abnormalities in fish embryos exposed to polycyclic aromatic hydrocarbons. Toxicol Appl Pharmacol. 2004;196(2):191-205.

36. Henry TR, Spitsbergen JM, Hornung MW, Abnet CC, Peterson RE. Early life stage toxicity of 2,3,7,8-tetrachlorodibenzo-p-dioxin in zebrafish (Danio rerio). Toxicol Appl Pharmacol. 1997;142(1):56-68.

37. Bonventre JA, White LA, Cooper KR. Craniofacial abnormalities and altered wnt and mmp mRNA expression in zebrafish embryos exposed to gasoline oxygenates ETBE and TAME. Aquatic Toxicol. 2012;120-121(0):45-53.

38. Mecke A, Majoros IJ, Patri AK, Baker JR Jr, Holl MM, Orr BG. Lipid bilayer disruption by polycationic polymers: the roles of size and chemical functional group. Langmuir. 2005;21(23):10348-10354.

39. Crooks RM, Zhao M, Sun L, Chechik V, Yeung LK. Dendrimerencapsulated metal nanoparticles: synthesis, characterization, and applications to catalysis. Acc Chem Res. 2001;34(3):181-190. 
40. Maiti PK, Çağın T, Wang G, Goddard WA. Structure of PAMAM dendrimers: generations 1 through 11. Macromolecules. 2004; 37(16):6236-6254.

41. El-Sayed M, Ginski M, Rhodes C, Ghandehari H. Transepithelial transport of poly(amidoamine) dendrimers across Caco-2 cell monolayers. J Control Release. 2002;81(3):355-365.

42. Frohlich E. The role of surface charge in cellular uptake and cytotoxicity of medical nanoparticles. Int J Nanomedicine. 2012;7:5577-5591.

43. Kitchens KM, El-Sayed MEH, Ghandehari H. Transepithelial and endothelial transport of poly(amidoamine) dendrimers. Adv Drug Deliv Rev. 2005;57(15):2163-2176.

44. McNerny DQ, Leroueil PR, Baker JR. Understanding specific and nonspecific toxicities: a requirement for the development of dendrimerbased pharmaceuticals. Wiley Interdiscip Rev Nanomed Nanobiotechnol. 2010;2(3):249-259.

45. Lee JH, Lim YB, Choi JS, Choi MU, Yang CH, Park JS. Quaternized polyamidoamine dendrimers as novel gene delivery system: relationship between degree of quaternization and their influences. Bull Korean Chem Soc. 2003;24(11):1637-1640.

46. Lee J-H, Cha KE, Kim MS, et al. Nanosized polyamidoamine (PAMAM) dendrimer-induced apoptosis mediated by mitochondrial dysfunction. Toxicol Lett. 2009;190(2):202-207.

47. Mukherjee SP, Byrne HJ. Polyamidoamine dendrimer nanoparticle cytotoxicity, oxidative stress, caspase activation and inflammatory response: experimental observation and numerical simulation. Nanomedicin. 2013;9(2):202-211.
48. Shukla D, Schneider CP, Trout BL. Effects of PAMAM dendrimer salt solutions on protein stability. J Phys Chem Lett. 2011;2(14): 1782-1788.

49. Kitchens K, Foraker A, Kolhatkar R, Swaan P, Ghandehari H. Endocytosis and interaction of poly(amidoamine) dendrimers with caco-2 cells. Pharm Res. 2007;24(11):2138-2145.

50. Thomas TP, Majoros I, Kotlyar A, Mullen D, Banaszak Holl MM, Baker JR. Cationic poly(amidoamine) dendrimer induces lysosomal apoptotic pathway at therapeutically relevant concentrations. Biomacromolecules. 2009;10(12):3207-3214.

51. Giri J, Diallo MS, Iii WAG, Dalleska NF, Fang X, Tang Y. Partitioning of poly(amidoamine) dendrimers between n-octanol and water. Environ Sci Technol. 2009;43(13):5123-5129.

52. Hong S, Bielinska AU, Mecke A, et al. Interaction of poly(amidoamine) dendrimers with supported lipid bilayers and cells: hole formation and the relation to transport. Bioconjug Chem. 2004;15(4):774-782.

53. Leroueil PR, Hong S, Mecke A, Baker JR, Orr BG, Banaszak Holl MM. Nanoparticle interaction with biological membranes: does nanotechnology present a janus face? Acc Cheml Res. 2007;40(5): 335-342.

54. Leroueil PR, Berry SA, Duthie K, et al. Wide varieties of cationic nanoparticles induce defects in supported lipid bilayers. Nano Letters. 2008;8(2):420-424.

55. Yang RSH, Garman RH, Maronpott RR, McKelvey JA, Weil CS, Woodside MD. Acute and subchronic toxicity of ethylenediamine in laboratory animals. Toxicol Sci. 1983;3(6):512-520. 


\section{Supplementary material}

Table SI Strength of correlation between the concentration and response

\begin{tabular}{ll}
\hline Dendrimer & Spearmen's P-value \\
\hline PAMAM G3-amine & $<0.001$ \\
PAMAM G4-amine & $<0.002$ \\
PAMAM G5-amine & $<0.002$ \\
PAMAM G6-amine & $<0.001$ \\
PAMAM G6-succinamic acid & 0.0212 \\
PAMAM G5-succinamic acid & 0.839 \\
PAMAM G6-amidoethanol & $<0.002$ \\
Thio G0.5-aldehyde & 0.29 \\
Thio GI.5-aldehyde & 0.578 \\
Thio G2.5-aldehyde & 0.794 \\
Thio G3.5-aldehyde & 0.29 \\
Thio G5-aldehyde & 0.794 \\
\hline
\end{tabular}

Abbreviations: PAMAM, polyamidoamine; Thio, thiophosphoryl; G, generation.

\section{Publish your work in this journal}

The International Journal of Nanomedicine is an international, peerreviewed journal focusing on the application of nanotechnology in diagnostics, therapeutics, and drug delivery systems throughout the biomedical field. This journal is indexed on PubMed Central, MedLine, CAS, SciSearch ${ }^{\circledR}$, Current Contents ${ }^{\circledR} /$ Clinical Medicine,

Journal Citation Reports/Science Edition, EMBase, Scopus and the Elsevier Bibliographic databases. The manuscript management system is completely online and includes a very quick and fair peer-review system, which is all easy to use. Visit http://www.dovepress.com/ testimonials.php to read real quotes from published authors. 